CRYSTALLOGRAPHIC COMMUNICATIONS

ISSN 2056-9890

Received 30 November 2016

Accepted 15 December 2016

Edited by C. Rizzoli, Universita degli Studi di Parma, Italy

Keywords: crystal structure; hydrogen bonds; isoxazolidines; nitrones; sulfones.

CCDC reference: 1519195

Supporting information: this article has supporting information at journals.iucr.org/e

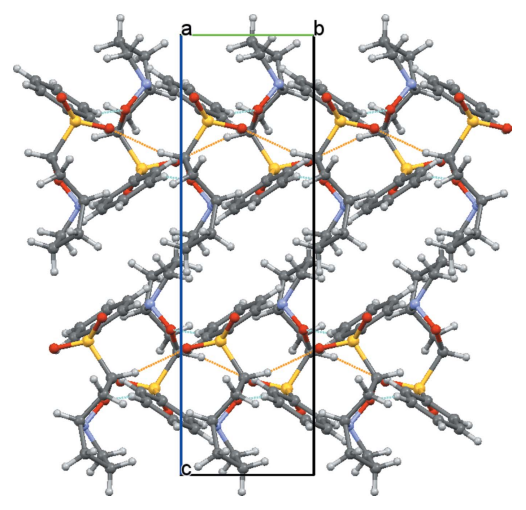

OPEN $\odot$ ACCESS

\section{Crystal structure of $\left(2 R^{*}, 3 \mathrm{a} R^{*}\right)$-2-phenylsulfonyl- 2,3,3a,4,5,6-hexahydropyrrolo[1,2-b]isoxazole}

\author{
Yaiza Hernández, ${ }^{a}$ Isidro Marcos, ${ }^{a}$ Narciso M. Garrido, ${ }^{a}$ Francisca Sanz ${ }^{b}$ and \\ David Diez ${ }^{\mathrm{a} *}$
}

\begin{abstract}
${ }^{a}$ Departamento de Química Orgánica, Universidad de Salamanca, Plaza de los Caidos, 37008 Salamanca, Spain, and bervicio de Difracción de Rayos X, Universidad de Salamanca, Plaza de los Caidos, 37008 Salamanca, Spain.

*Correspondence e-mail: ddm@usal.es
\end{abstract}

The title compound, $\mathrm{C}_{12} \mathrm{H}_{15} \mathrm{NO}_{3} \mathrm{~S}$, was prepared by 1,3-dipolar cycloaddition of 3,4-dihydro- $2 H$-pyrrole 1-oxide and phenyl vinyl sulfone. In the molecule, both fused five-membered rings display a twisted conformation. In the crystal, C$\mathrm{H}$. . O hydrogen bonds link neighbouring molecules, forming chains running parallel to the $b$ axis.

\section{Chemical context}

1,3-Dipolar cycloaddition is one of the most useful reaction in organic synthesis (Pellissier, 2007). Nitrones have been used in the synthesis of many kinds of isoxazolidines (Falkowska et al., 2015 ) by 1,3-dipolar cycloaddition of nitrones with sulfones (Flores, García, Garrido, Nieto et al., 2012) and have demonstrate a range of biological activities including antibiotic, gene expression regulation and cancer cell cytotoxicity (Karyakarte et al., 2012). Our research group is interested in the synthesis of isoxazolidines such as the title compound, for application in organic synthesis (Flores et al., 2011a,b; Flores, García-García et al., 2012; Flores, García, Garrido, Sanz et al., 2012; Flores et al., 2013).<smiles>[O-][N+]1=CCCC1</smiles>

I

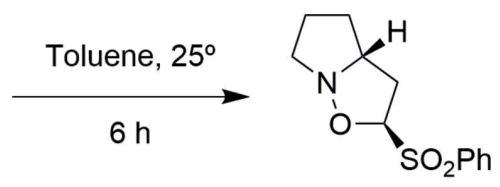

III

\section{Structural commentary}

The molecular structure of the title compound, which consists of an anisoxazol derivative with a phenyl sulfone group as substituent, is shown in Fig. 1. Both the fused five-membered rings assume a twist conformation, as indicated by puckering parameters $Q=0.338$ (3) $\AA, \varphi=-73.5(7)^{\circ}$ for the pyrrole ring and $Q=0.209$ (2) $\AA, \varphi=-97.5(6)^{\circ}$ for the isoxazole ring. The dihedral angle between the mean planes of the five-membered rings is $64.91(10)^{\circ}$. All the bond lengths are within normal ranges. The $\mathrm{C}-\mathrm{S}-\mathrm{C}$ and $\mathrm{O}-\mathrm{S}-\mathrm{O}$ angles are 104.34 (9) and $118.54(11)^{\circ}$, respectively. The large $\mathrm{O}-\mathrm{S}-\mathrm{O}$ angle, and its deviation from the ideal $109.5^{\circ}$ angle, can be explained by the repulsion of the lone pairs of the oxygen atoms as far away from each other as possible minimizing the $\mathrm{C}-\mathrm{S}-\mathrm{C}$ angle. The $\mathrm{C} 5-\mathrm{C} 6-\mathrm{S} 1-\mathrm{C} 7$ torsion angle is $171.26(15)^{\circ}$. 


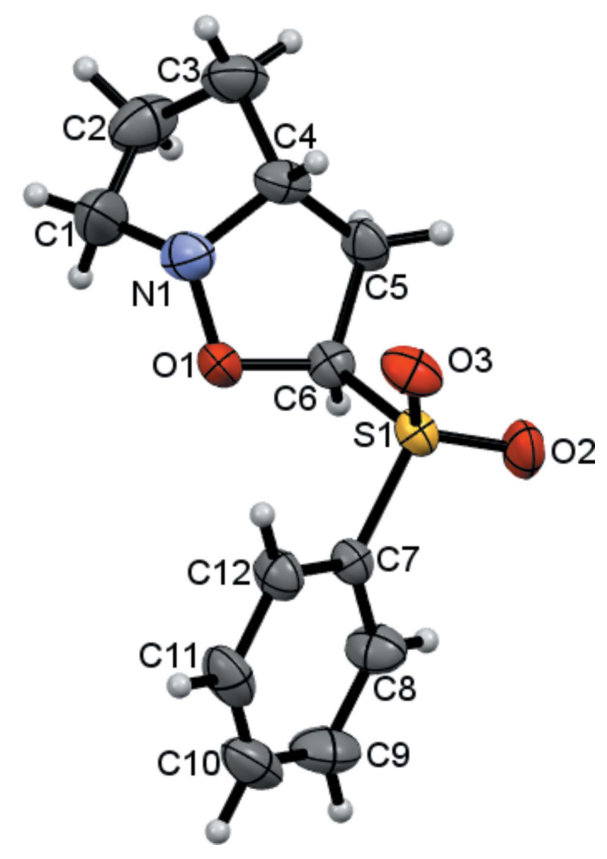

Figure 1

The molecular structure of the title compound, with displacement ellipsoids drawn at the $50 \%$ probability level. $\mathrm{H}$ atoms are shown as spheres of arbitrary radius.

\section{Supramolecular features}

In the extended structure of the title compound, intermolecular $\mathrm{C}-\mathrm{H} \cdots \mathrm{O}$ hydrogen bonds involving the $\mathrm{O} 1$ isoxazole and the $\mathrm{O} 3$ phenyl sulfone $\mathrm{O}$ atoms as donors (Table 1) lead to molecular chains running parallel to the $b$ axis (Fig. 2).

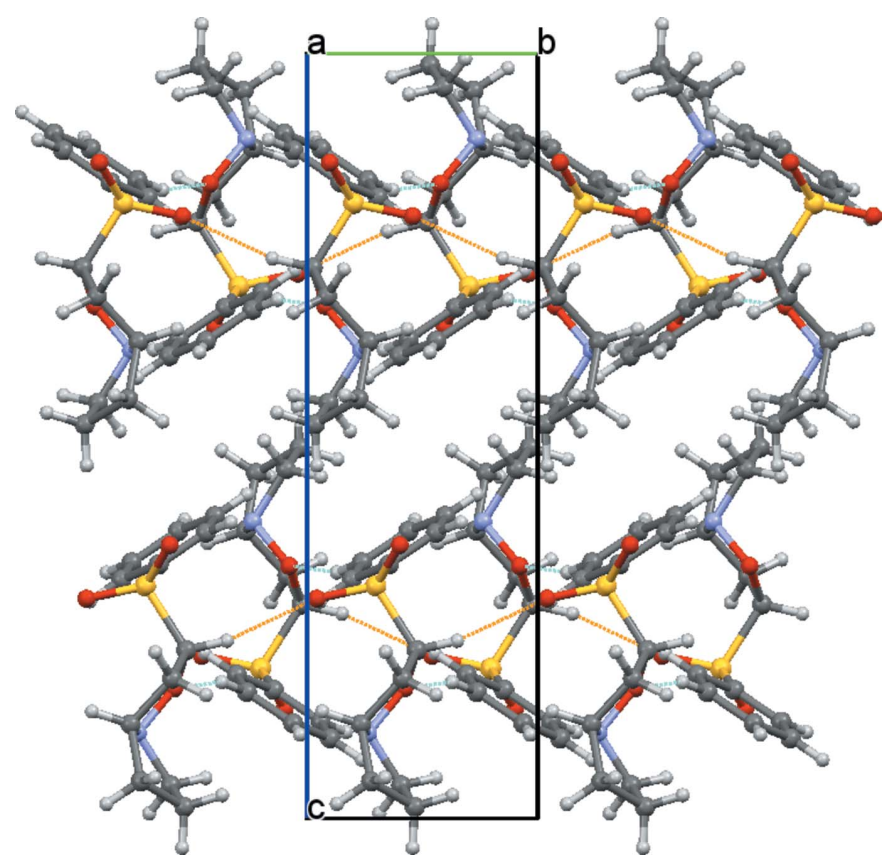

Figure 2

Crystal packing of the title compound viewed along the [100] direction, showing intermolecular hydrogen bonding (dashed lines).
Table 1

Hydrogen-bond geometry $\left(\AA{ }^{\circ}\right)$.

\begin{tabular}{lllll}
\hline$D-\mathrm{H} \cdots A$ & $D-\mathrm{H}$ & $\mathrm{H} \cdots A$ & $D \cdots A$ & $D-\mathrm{H} \cdots A$ \\
\hline $\mathrm{C} 6-\mathrm{H} 6 \cdots \mathrm{O}^{\mathrm{i}}$ & 0.98 & 2.35 & $3.314(3)$ & 168 \\
$\mathrm{C}^{1} 1-\mathrm{H} 11 \cdots{ }^{\mathrm{ii}}$ & 0.93 & 2.49 & $3.364(3)$ & 157 \\
\hline
\end{tabular}

Symmetry codes: (i) $x, y-1, z$; (ii) $-x+1, y+\frac{1}{2},-z+\frac{1}{2}$.

\section{Synthesis and crystallization}

In the synthesis, $5 \mathrm{~g}$ of phenyl vinyl sulfone (II) $(29.40 \mathrm{mmol})$ was added to a solution of $2 \mathrm{~g}$ of 3,4-dihydro- $2 \mathrm{H}$-pyrrole 1 oxide (I) $(23.50 \mathrm{mmol})$ in toluene $(75 \mathrm{~mL})$ at room temperature. The resulting mixture was stirred for $6 \mathrm{~h}$, then it was quenched with a saturated aqueous solution of $\mathrm{NH}_{4} \mathrm{Cl}$ and the product was extracted with EtOAc. The combined organic layers were washed with brine, dried over $\mathrm{Na}_{2} \mathrm{SO}_{4}$, filtered, and concentrated, yielding the crude product (III) $(8.93 \mathrm{mmol}$, $38 \%)$. The resulting crude residue was purified by flash chromatography (silica gel, hexane/EtOAc 6:4 v/v) and crystallized from hexane/ethyl acetate solution. IR (film): 3436 (broad), 3068, 2946, 2868, 1442, 1377, 1307, 1148, $1074 \mathrm{~cm}^{-1}$. ${ }^{1} \mathrm{H}$ NMR (400 MHz, $\mathrm{CDCl}_{3}, \delta$ p.p.m.): $7.99(2 \mathrm{H}, d, J=8.0 \mathrm{~Hz}$, Hortho $), 7.70(1 \mathrm{H}, t, J=7.9 \mathrm{~Hz}, \mathrm{H}$ para $), 7.58(2 \mathrm{H}, t, J=8.0 \mathrm{~Hz}$, Hmeta), $5.04(1 \mathrm{H}, d d, J=4.0$ y $8.4 \mathrm{~Hz}, \mathrm{H}-2), 3.85-3.81(1 \mathrm{H}, m$, H-3a), 3.36-3.31 (1H, $\left.m, \mathrm{H}_{\mathrm{B}}-6\right), 3.23(1 \mathrm{H}, d d d, J=4.0,7.0$ y $\left.12.4 \mathrm{~Hz}, \mathrm{H}_{\mathrm{B}}-3\right), 3.05\left(1 \mathrm{H}, d t, J=8.3\right.$ y $\left.13.8 \mathrm{~Hz}, \mathrm{H}_{\mathrm{A}}-6\right), 2.50(1 \mathrm{H}$, $d d d, J=4.0,8.4$ y $\left.12.4 \mathrm{~Hz}, \mathrm{H}_{\mathrm{A}}-3\right), 2.04-1.93\left(2 \mathrm{H}, m, \mathrm{H}_{\mathrm{A}}-4\right.$ у $\left.\mathrm{H}_{\mathrm{A}}-5\right)$, 1.76-1.74 (1H, $\left.m, \mathrm{H}_{\mathrm{B}}-5\right)$, 1.60-1.57 (1H, $\left.m, \mathrm{H}_{\mathrm{B}}-4\right)$.

Table 2

Experimental details.

\begin{tabular}{ll}
\hline Crystal data & \\
Chemical formula & $\mathrm{C}_{12} \mathrm{H}_{15} \mathrm{NO}_{3} \mathrm{~S}$ \\
$M_{\mathrm{r}}$ & 253.31 \\
Crystal system, space group & Monoclinic, $P 2_{1} / c$ \\
Temperature (K) & 298 \\
$a, b, c(\AA)$ & $12.5730(4), 5.4443(2), 18.2266(6)$ \\
$\beta\left({ }^{\circ}\right.$ & $97.754(2)$ \\
$V\left(\AA^{3}\right)$ & $1236.22(7)$ \\
$Z$ & 4 \\
Radiation type & $\mathrm{Cu} K \alpha$ \\
$\mu\left(\mathrm{mm}^{-1}\right)$ & 2.31 \\
Crystal size $(\mathrm{mm})$ & $0.25 \times 0.20 \times 0.10$ \\
& \\
Data collection & Bruker APEXII CCD area \\
Diffractometer & detector \\
& Multi-scan $(S A D A B S ;$ Bruker, \\
Absorption correction & $2006)$ \\
& $0.603,0.794$ \\
$T_{\text {min }}, T_{\text {max }}$ & $9571,2074,1949$ \\
No. of measured, independent and & \\
$\quad$ observed $[I>2 \sigma(I)]$ reflections & 0.032 \\
$R_{\text {int }}$ & 0.596 \\
$(\text { sin } \theta / \lambda)_{\text {max }}\left(\AA^{-1}\right)$ & \\
Refinement & \\
$R\left[F^{2}>2 \sigma\left(F^{2}\right)\right], w R\left(F^{2}\right), S$ & $0.042,0.109,1.04$ \\
No. of reflections & 2074 \\
No. of parameters & 154 \\
$H$-atom treatment & H-atom parameters constrained \\
$\Delta \rho_{\text {max }}, \Delta \rho_{\text {min }}\left(\mathrm{e} \AA^{-3}\right)$ & $0.23,-0.25$ \\
\hline & \\
&
\end{tabular}

Computer programs: APEX2 and SAINT (Bruker 2006), SHELXS97, SHELXL97 and SHELXTL/PC (Sheldrick, 2008) and Mercury (Macrae et al., 2006). 
${ }^{13} \mathrm{CNMR}$ (100 MHz, $\mathrm{CDCl}_{3} \delta$ p.p.m.): 136.7 (C-ipso), 133.9 $\left(\mathrm{CH}_{\text {para }}\right), 129.5\left(2 \mathrm{CH}_{\text {meta }}\right), 128.9\left(2 \mathrm{CH}_{\text {ortho }}\right), 92.5(\mathrm{CH}-2), 65.5$ (CH-3a), $57.3\left(\mathrm{CH}_{2}-6\right), 36.8\left(\mathrm{CH}_{2}-3\right), 30.8\left(\mathrm{CH}_{2}-4\right), 23.8\left(\mathrm{CH}_{2}-\right.$ 5). HRMS (EI): $\mathrm{C}_{12} \mathrm{H}_{15} \mathrm{NO}_{3} \mathrm{NaS}$ requires $(M+\mathrm{Na})^{+}, 276.0665$, found 276.0682 .

\section{Refinement}

Crystal data, data collection and structure refinement details are summarized in Table 2. The hydrogen atoms were positioned geometrically, with $\mathrm{C}-\mathrm{H}$ distances constrained to $0.93 \AA$ (aromatic CH), $0.97 \AA$ (methylene $\mathrm{CH}_{2}$ ), 0.98 (methyne $\mathrm{CH}$ ) and refined using a riding mode with $U_{\text {iso }}(\mathrm{H})=$ $1.2 U_{\text {eq }}(\mathrm{C})$.

\section{Acknowledgements}

The authors gratefully acknowledge help from A. Lithgow (NMR) and C. Raposo (MS) of the Universidad de Salamanca.

\section{Funding information}

Funding for this research was provided by: MINECO (award Nos. CTQ2015-68175-R, SAF2014-59716-R); FEDER (award No. Junta de Castilla y León UCI21).

\section{References}

Bruker (2006). APEX2, SAINT and SADABS. Bruker AXS Inc., Madison, Wisconsin, USA.

Falkowska, E., Laurent, M. Y., Tognetti, V., Joubert, L., Jubault, P., Bouillon, J.-P. \& Pannecoucke, X. (2015). Tetrahedron, 71, $8067-$ 8076.

Flores, M., García, P., Garrido, N. M., Nieto, C. T., Basabe, P., Marcos, I. S., Sanz-González, F., Goodman, J. M. \& Díez, D. (2012). Tetrahedron Asymmetry, 23, 76-85.

Flores, M., García-García, P., Garrido, N. M., Marcos, I. S., Sanz, F. \& Díez, D. (2012). RSC Adv. 2, 11040-11048.

Flores, M., García-García, P., Garrido, N. M., Marcos, I. S., SanzGonzález, F. \& Díez, D. (2013). J. Org. Chem. 78, 7068-7075.

Flores, M. F., Garcia, P., Garrido, N. M., Sanz, F. \& Diez, D. (2011a). Acta Cryst. E67, o1115.

Flores, M. F., Garcia, P., Garrido, N. M., Sanz, F. \& Diez, D. (2011b). Acta Cryst. E67, o1116-o1117.

Flores, M. F., Garcia, P., Garrido, N. M., Sanz, F. \& Diez, D. (2012). Acta Cryst. E68, o2560.

Karyakarte, S. D., Smith, T. P. \& Chemler, S. R. (2012). J. Org. Chem. 77, 7755-7760.

Macrae, C. F., Edgington, P. R., McCabe, P., Pidcock, E., Shields, G. P., Taylor, R., Towler, M. \& van de Streek, J. (2006). J. Appl. Cryst. 39, 453-457.

Pellissier, H. (2007). Tetrahedron, 63, 3235-3285.

Sheldrick, G. M. (2008). Acta Cryst. A64, 112-122. 


\section{supporting information}

Acta Cryst. (2017). E73, 85-87 [https://doi.org/10.1107/S2056989016019952]

Crystal structure of $\left(2 R^{*}, 3 \mathrm{a} R^{*}\right)$-2-phenylsulfonyl-2,3,3a,4,5,6-hexahydropyrrolo$[1,2-b]$ isoxazole

Yaiza Hernández, Isidro Marcos, Narciso M. Garrido, Francisca Sanz and David Diez

Computing details

Data collection: APEX2 (Bruker 2006); cell refinement: SAINT (Bruker 2006); data reduction: SAINT (Bruker 2006);

program(s) used to solve structure: SHELXS97 (Sheldrick, 2008); program(s) used to refine structure: SHELXL97

(Sheldrick, 2008); molecular graphics: Mercury (Macrae et al., 2006); software used to prepare material for publication:

SHELXTL/PC (Sheldrick, 2008).

$\left(2 R^{*}, 3 \mathrm{a} R^{*}\right)$-2-Phenylsulfonyl-2,3,3a,4,5,6-hexahydropyrrolo[1,2-b] isoxazole

Crystal data

$\mathrm{C}_{12} \mathrm{H}_{15} \mathrm{NO}_{3} \mathrm{~S}$

$M_{r}=253.31$

Monoclinic, $P 2{ }_{1} / c$

Hall symbol: -P 2ybc

$a=12.5730(4) \AA$

$b=5.4443(2) \AA$

$c=18.2266(6) \AA$

$\beta=97.754(2)^{\circ}$

$V=1236.22(7) \AA^{3}$

$Z=4$

$F(000)=536$

$D_{\mathrm{x}}=1.361 \mathrm{Mg} \mathrm{m}^{-3}$

$\mathrm{Cu} K \alpha$ radiation, $\lambda=1.54178 \AA$

Cell parameters from 1548 reflections

$\theta=8.7-66.1^{\circ}$

$\mu=2.31 \mathrm{~mm}^{-1}$

$T=298 \mathrm{~K}$

Prismatic, colorless

Data collection

Bruker APEXII CCD area detector

diffractometer

Radiation source: fine-focus sealed tube

$0.25 \times 0.20 \times 0.10 \mathrm{~mm}$

Graphite monochromator

phi and $\omega$ scans

Absorption correction: multi-scan

(SADABS; Bruker, 2006)

$T_{\min }=0.603, T_{\max }=0.794$

9571 measured reflections

2074 independent reflections

1949 reflections with $I>2 \sigma(I)$

$R_{\text {int }}=0.032$

$\theta_{\text {max }}=66.8^{\circ}, \theta_{\text {min }}=8.5^{\circ}$

$h=-14 \rightarrow 13$

$k=-6 \rightarrow 6$

$l=-17 \rightarrow 21$

Refinement

Refinement on $F^{2}$

Least-squares matrix: full

$R\left[F^{2}>2 \sigma\left(F^{2}\right)\right]=0.042$

$w R\left(F^{2}\right)=0.109$

$S=1.04$

2074 reflections

154 parameters

0 restraints

Primary atom site location: structure-invariant direct methods

Secondary atom site location: difference Fourier map

Hydrogen site location: inferred from neighbouring sites

$\mathrm{H}$-atom parameters constrained 
$w=1 /\left[\sigma^{2}\left(F_{\mathrm{o}}^{2}\right)+(0.048 P)^{2}+0.5869 P\right]$

where $P=\left(F_{\mathrm{o}}^{2}+2 F_{\mathrm{c}}^{2}\right) / 3$

$(\Delta / \sigma)_{\max }<0.001$

$$
\Delta \rho_{\max }=0.23 \mathrm{e} \AA^{-3}
$$

$\Delta \rho_{\min }=-0.25$ e $\AA^{-3}$

\section{Special details}

Geometry. All esds (except the esd in the dihedral angle between two 1.s. planes) are estimated using the full covariance matrix. The cell esds are taken into account individually in the estimation of esds in distances, angles and torsion angles; correlations between esds in cell parameters are only used when they are defined by crystal symmetry. An approximate (isotropic) treatment of cell esds is used for estimating esds involving l.s. planes.

Refinement. Refinement of $\mathrm{F}^{2}$ against ALL reflections. The weighted R-factor $\mathrm{wR}$ and goodness of fit $\mathrm{S}$ are based on $\mathrm{F}^{2}$, conventional R-factors $\mathrm{R}$ are based on $\mathrm{F}$, with $\mathrm{F}$ set to zero for negative $\mathrm{F}^{2}$. The threshold expression of $\mathrm{F}^{2}>2 \operatorname{sigma}\left(\mathrm{F}^{2}\right)$ is used only for calculating R-factors(gt) etc. and is not relevant to the choice of reflections for refinement. R-factors based on $\mathrm{F}^{2}$ are statistically about twice as large as those based on F, and R- factors based on ALL data will be even larger.

Fractional atomic coordinates and isotropic or equivalent isotropic displacement parameters $\left(\AA^{2}\right)$

\begin{tabular}{lllll}
\hline & $x$ & $y$ & $z$ & $U_{\text {iso }} / U_{\text {eq }}$ \\
\hline S1 & $0.18509(4)$ & $0.20282(9)$ & $0.19580(3)$ & $0.0506(2)$ \\
O1 & $0.27595(12)$ & $0.0779(4)$ & $0.33002(8)$ & $0.0780(5)$ \\
O2 & $0.10289(12)$ & $0.0979(4)$ & $0.14263(9)$ & $0.0745(5)$ \\
O3 & $0.17911(13)$ & $0.4582(3)$ & $0.21257(10)$ & $0.0722(5)$ \\
N1 & $0.24857(16)$ & $0.2358(4)$ & $0.38806(12)$ & $0.0767(6)$ \\
C1 & $0.2723(3)$ & $0.0922(13)$ & $0.45671(19)$ & $0.173(3)$ \\
H1A & 0.3095 & -0.0579 & 0.4469 & $0.207^{*}$ \\
H1B & 0.3185 & 0.1863 & 0.4933 & $0.207^{*}$ \\
C2 & $0.1731(3)$ & $0.0332(7)$ & $0.48481(17)$ & $0.1044(11)$ \\
H2A & 0.1821 & 0.0422 & 0.5384 & $0.125^{*}$ \\
H2B & 0.1487 & -0.1303 & 0.4696 & $0.125^{*}$ \\
C3 & $0.0964(3)$ & $0.2227(6)$ & $0.45154(17)$ & $0.0932(9)$ \\
H3A & 0.1018 & 0.3717 & 0.4810 & $0.112^{*}$ \\
H3B & 0.0231 & 0.1629 & 0.4466 & $0.112^{*}$ \\
C4 & $0.13178(19)$ & $0.2665(5)$ & $0.37671(14)$ & $0.0653(6)$ \\
H4 & 0.1134 & 0.4343 & 0.3602 & $0.078^{*}$ \\
C5 & $0.08854(17)$ & $0.0856(5)$ & $0.31715(13)$ & $0.0669(6)$ \\
H5A & 0.0605 & -0.0600 & 0.3385 & $0.080^{*}$ \\
H5B & 0.0322 & 0.1592 & 0.2824 & $0.080^{*}$ \\
C6 & $0.18549(16)$ & $0.0244(4)$ & $0.27984(11)$ & $0.0548(5)$ \\
H6 & 0.1848 & -0.1510 & 0.2677 & $0.066^{*}$ \\
C7 & $0.31162(15)$ & $0.1448(4)$ & $0.16793(11)$ & $0.0489(5)$ \\
C8 & $0.3261(2)$ & $-0.0590(5)$ & $0.12636(15)$ & $0.0748(7)$ \\
H8 & 0.2699 & -0.1680 & 0.1129 & $0.090^{*}$ \\
C9 & $0.4260(3)$ & $-0.0996(6)$ & $0.10473(19)$ & $0.0949(9)$ \\
H9 & 0.4371 & -0.2375 & 0.0766 & $0.114^{*}$ \\
C10 & $0.5086(2)$ & $0.0610(7)$ & $0.12436(18)$ & $0.0902(9)$ \\
H10 & 0.5754 & 0.0320 & 0.1095 & $0.108^{*}$ \\
C11 & $0.4932(2)$ & $0.2622(6)$ & $0.16530(18)$ & $0.0843(8)$ \\
H11 & 0.5495 & 0.3712 & 0.1783 & $0.101^{*}$ \\
C12 & $0.39431(18)$ & $0.3066(4)$ & $0.18785(14)$ & $0.0650(6)$ \\
H12 & 0.3839 & 0.4444 & 0.2162 & $0.078^{*}$ \\
& & & &
\end{tabular}


Atomic displacement parameters $\left(\AA^{2}\right)$

\begin{tabular}{lllllll}
\hline & $U^{11}$ & $U^{22}$ & $U^{33}$ & $U^{12}$ & $U^{13}$ & $U^{23}$ \\
\hline S1 & $0.0409(3)$ & $0.0501(3)$ & $0.0601(3)$ & $-0.00016(19)$ & $0.0047(2)$ & $0.0026(2)$ \\
O1 & $0.0465(8)$ & $0.1268(15)$ & $0.0598(9)$ & $0.0238(9)$ & $0.0034(7)$ & $-0.0082(10)$ \\
O2 & $0.0485(8)$ & $0.1031(13)$ & $0.0677(9)$ & $-0.0149(8)$ & $-0.0079(7)$ & $0.0020(9)$ \\
O3 & $0.0706(10)$ & $0.0466(8)$ & $0.1034(12)$ & $0.0115(7)$ & $0.0265(9)$ & $0.0072(8)$ \\
N1 & $0.0565(12)$ & $0.0980(16)$ & $0.0779(13)$ & $-0.0213(11)$ & $0.0169(10)$ & $-0.0249(12)$ \\
C1 & $0.102(3)$ & $0.350(8)$ & $0.0652(18)$ & $0.079(4)$ & $0.0086(18)$ & $0.015(3)$ \\
C2 & $0.146(3)$ & $0.098(2)$ & $0.0722(17)$ & $0.015(2)$ & $0.0272(19)$ & $0.0106(16)$ \\
C3 & $0.094(2)$ & $0.111(2)$ & $0.0831(18)$ & $0.0098(18)$ & $0.0415(17)$ & $0.0043(17)$ \\
C4 & $0.0646(14)$ & $0.0611(13)$ & $0.0743(14)$ & $0.0105(11)$ & $0.0250(11)$ & $0.0033(11)$ \\
C5 & $0.0437(11)$ & $0.0849(16)$ & $0.0728(14)$ & $-0.0104(11)$ & $0.0101(10)$ & $0.0128(12)$ \\
C6 & $0.0541(11)$ & $0.0495(11)$ & $0.0603(12)$ & $-0.0008(9)$ & $0.0062(9)$ & $-0.0008(9)$ \\
C7 & $0.0446(10)$ & $0.0478(11)$ & $0.0539(10)$ & $-0.0008(8)$ & $0.0054(8)$ & $0.0021(9)$ \\
C8 & $0.0772(16)$ & $0.0601(14)$ & $0.0901(17)$ & $-0.0040(12)$ & $0.0223(13)$ & $-0.0145(13)$ \\
C9 & $0.104(2)$ & $0.0832(19)$ & $0.105(2)$ & $0.0252(18)$ & $0.0439(18)$ & $-0.0077(17)$ \\
C10 & $0.0640(16)$ & $0.108(2)$ & $0.105(2)$ & $0.0248(16)$ & $0.0352(15)$ & $0.0315(19)$ \\
C11 & $0.0447(13)$ & $0.100(2)$ & $0.108(2)$ & $-0.0088(13)$ & $0.0087(13)$ & $0.0152(17)$ \\
C12 & $0.0512(12)$ & $0.0650(14)$ & $0.0782(15)$ & $-0.0091(10)$ & $0.0064(11)$ & $-0.0057(11)$ \\
& & & & & &
\end{tabular}

Geometric parameters $(\AA, \stackrel{\circ}{)})$

\begin{tabular}{llll}
\hline $\mathrm{S} 1-\mathrm{O} 3$ & $1.4277(16)$ & $\mathrm{C} 4-\mathrm{C} 5$ & $1.511(4)$ \\
$\mathrm{S} 1-\mathrm{O} 2$ & $1.4364(16)$ & $\mathrm{C} 4-\mathrm{H} 4$ & 0.9800 \\
$\mathrm{~S} 1-\mathrm{C} 7$ & $1.763(2)$ & $\mathrm{C} 5-\mathrm{C} 6$ & $1.511(3)$ \\
$\mathrm{S} 1-\mathrm{C} 6$ & $1.813(2)$ & $\mathrm{C} 5-\mathrm{H} 5 \mathrm{~A}$ & 0.9700 \\
$\mathrm{O} 1-\mathrm{C} 6$ & $1.391(3)$ & $\mathrm{C} 5-\mathrm{H} 5 \mathrm{~B}$ & 0.9700 \\
$\mathrm{O} 1-\mathrm{N} 1$ & $1.440(3)$ & $\mathrm{C} 6-\mathrm{H} 6$ & 0.9800 \\
$\mathrm{~N} 1-\mathrm{C} 4$ & $1.465(3)$ & $\mathrm{C} 7-\mathrm{C} 8$ & $1.370(3)$ \\
$\mathrm{N} 1-\mathrm{C} 1$ & $1.472(5)$ & $\mathrm{C} 7-\mathrm{C} 12$ & $1.374(3)$ \\
$\mathrm{C} 1-\mathrm{C} 2$ & $1.446(5)$ & $\mathrm{C} 8-\mathrm{C} 9$ & $1.384(4)$ \\
$\mathrm{C} 1-\mathrm{H} 1 \mathrm{~A}$ & 0.9700 & $\mathrm{C} 8-\mathrm{H} 8$ & 0.9300 \\
$\mathrm{C} 1-\mathrm{H} 1 \mathrm{~B}$ & 0.9700 & $\mathrm{C} 9-\mathrm{C} 10$ & $1.367(5)$ \\
$\mathrm{C} 2-\mathrm{C} 3$ & $1.485(4)$ & $\mathrm{C} 9-\mathrm{H} 9$ & 0.9300 \\
$\mathrm{C} 2-\mathrm{H} 2 \mathrm{~A}$ & 0.9700 & $\mathrm{C} 10-\mathrm{C} 11$ & $1.354(5)$ \\
$\mathrm{C} 2-\mathrm{H} 2 \mathrm{~B}$ & 0.9700 & $\mathrm{C} 10-\mathrm{H} 10$ & 0.9300 \\
$\mathrm{C} 3-\mathrm{C} 4$ & $1.510(4)$ & $\mathrm{C} 11-\mathrm{C} 12$ & $1.382(4)$ \\
$\mathrm{C} 3-\mathrm{H} 3 \mathrm{~A}$ & 0.9700 & $\mathrm{C} 11-\mathrm{H} 11$ & 0.9300 \\
$\mathrm{C} 3-\mathrm{H} 3 \mathrm{~B}$ & 0.9700 & $\mathrm{C} 12-\mathrm{H} 12$ & \\
& & & 109.8 \\
$\mathrm{O} 3-\mathrm{S} 1-\mathrm{O} 2$ & $118.54(11)$ & $\mathrm{C} 3-\mathrm{C} 4-\mathrm{H} 4$ & 109.8 \\
$\mathrm{O} 3-\mathrm{S} 1-\mathrm{C} 7$ & $108.17(9)$ & $\mathrm{C} 5-\mathrm{C} 4-\mathrm{H} 4$ & $103.48(17)$ \\
$\mathrm{O} 2-\mathrm{S} 1-\mathrm{C} 7$ & $109.24(10)$ & $\mathrm{C} 6-\mathrm{C} 5-\mathrm{C} 4$ & 111.1 \\
$\mathrm{O} 3-\mathrm{S} 1-\mathrm{C} 6$ & $109.58(10)$ & $\mathrm{C} 6-\mathrm{C} 5-\mathrm{H} 5 \mathrm{~A}$ & 111.1 \\
$\mathrm{O} 2-\mathrm{S} 1-\mathrm{C} 6$ & $106.06(10)$ & $\mathrm{C} 4-\mathrm{C} 5-\mathrm{H} 5 \mathrm{~A}$ & 111.1 \\
$\mathrm{C} 7-\mathrm{S} 1-\mathrm{C} 6$ & $104.34(9)$ & $\mathrm{C} 6-\mathrm{C} 5-\mathrm{H} 5 \mathrm{~B}$ & 111.1 \\
$\mathrm{C} 6-\mathrm{O} 1-\mathrm{N} 1$ & $110.65(15)$ & $\mathrm{C} 4-\mathrm{C} 5-\mathrm{H} 5 \mathrm{~B}$ &
\end{tabular}




$\begin{array}{llll}\mathrm{O} 1-\mathrm{N} 1-\mathrm{C} 4 & 107.39(17) & \mathrm{H} 5 \mathrm{~A}-\mathrm{C} 5-\mathrm{H} 5 \mathrm{~B} & 109.0 \\ \mathrm{O} 1-\mathrm{N} 1-\mathrm{C} 1 & 105.4(3) & \mathrm{O} 1-\mathrm{C} 6-\mathrm{C} 5 & 107.19(17) \\ \mathrm{C} 4-\mathrm{N} 1-\mathrm{C} 1 & 105.4(2) & \mathrm{O} 1-\mathrm{C} 6-\mathrm{S} 1 & 110.63(15) \\ \mathrm{C} 2-\mathrm{C} 1-\mathrm{N} 1 & 109.5(3) & \mathrm{C} 5-\mathrm{C} 6-\mathrm{S} 1 & 110.59(15) \\ \mathrm{C} 2-\mathrm{C} 1-\mathrm{H} 1 \mathrm{~A} & 109.8 & \mathrm{O} 1-\mathrm{C} 6-\mathrm{H} 6 & 109.5 \\ \mathrm{~N} 1-\mathrm{C} 1-\mathrm{H} 1 \mathrm{~A} & 109.8 & \mathrm{C} 5-\mathrm{C} 6-\mathrm{H} 6 & 109.5 \\ \mathrm{C} 2-\mathrm{C} 1-\mathrm{H} 1 \mathrm{~B} & 109.8 & \mathrm{~S} 1-\mathrm{C} 6-\mathrm{H} 6 & 120.9(2) \\ \mathrm{N} 1-\mathrm{C} 1-\mathrm{H} 1 \mathrm{~B} & 109.8 & \mathrm{C} 8-\mathrm{C} 7-\mathrm{C} 12 & 119.84(17) \\ \mathrm{H} 1 \mathrm{~A}-\mathrm{C} 1-\mathrm{H} 1 \mathrm{~B} & 108.2 & \mathrm{C} 8-\mathrm{C} 7-\mathrm{S} 1 & 119.31(16) \\ \mathrm{C} 1-\mathrm{C} 2-\mathrm{C} 3 & 104.2(3) & \mathrm{C} 12-\mathrm{C} 7-\mathrm{S} 1 & 118.8(3) \\ \mathrm{C} 1-\mathrm{C} 2-\mathrm{H} 2 \mathrm{~A} & 110.9 & \mathrm{C} 7-\mathrm{C} 8-\mathrm{C} 9 & 120.6 \\ \mathrm{C} 3-\mathrm{C} 2-\mathrm{H} 2 \mathrm{~A} & 110.9 & \mathrm{C} 7-\mathrm{C} 8-\mathrm{H} 8 & 120.6 \\ \mathrm{C} 1-\mathrm{C} 2-\mathrm{H} 2 \mathrm{~B} & 110.9 & \mathrm{C} 9-\mathrm{C} 8-\mathrm{H} 8 & 119.7 \\ \mathrm{C} 3-\mathrm{C} 2-\mathrm{H} 2 \mathrm{~B} & 110.9 & \mathrm{C} 10-\mathrm{C} 9-\mathrm{C} 8 & 119.7 \\ \mathrm{H} 2 \mathrm{~A}-\mathrm{C} 2-\mathrm{H} 2 \mathrm{~B} & 108.9 & \mathrm{C} 10-\mathrm{C} 9-\mathrm{H} 9 & 120.1(2) \\ \mathrm{C} 2-\mathrm{C} 3-\mathrm{C} 4 & 103.0(2) & \mathrm{C} 8-\mathrm{C} 9-\mathrm{H} 9 & 119.9 \\ \mathrm{C} 2-\mathrm{C} 3-\mathrm{H} 3 \mathrm{~A} & 111.2 & \mathrm{C} 11-\mathrm{C} 10-\mathrm{C} 9 & 119.9 \\ \mathrm{C} 4-\mathrm{C} 3-\mathrm{H} 3 \mathrm{~A} & 111.2 & \mathrm{C} 11-\mathrm{C} 10-\mathrm{H} 10 & 120.4(3) \\ \mathrm{C} 2-\mathrm{C} 3-\mathrm{H} 3 \mathrm{~B} & 111.2 & \mathrm{C} 9-\mathrm{C} 10-\mathrm{H} 10 & 119.8 \\ \mathrm{C} 4-\mathrm{C} 3-\mathrm{H} 3 \mathrm{~B} & 111.2 & \mathrm{C} 10-\mathrm{C} 11-\mathrm{C} 12 & 119.8 \\ \mathrm{H} 3 \mathrm{~A}-\mathrm{C} 3-\mathrm{H} 3 \mathrm{~B} & 109.1 & \mathrm{C} 10-\mathrm{C} 11-\mathrm{H} 11 & 119.2(2) \\ \mathrm{N} 1-\mathrm{C} 4-\mathrm{C} 3 & 105.5(2) & \mathrm{C} 12-\mathrm{C} 11-\mathrm{H} 11 & 120.4 \\ \mathrm{~N} 1-\mathrm{C} 4-\mathrm{C} 5 & 106.48(18) & \mathrm{C} 7-\mathrm{C} 12-\mathrm{C} 11 & 120.4 \\ \mathrm{C} 3-\mathrm{C} 4-\mathrm{C} 5 & 115.1(2) & \mathrm{C} 7-\mathrm{C} 12-\mathrm{H} 12 & \\ \text { N1-C4-H4 } & 109.8 & \mathrm{C} 11-\mathrm{C} 12-\mathrm{H} 12 & \\ & & & \end{array}$

Hydrogen-bond geometry $\left(\AA,{ }^{\circ}\right)$

\begin{tabular}{lllll}
\hline$D-\mathrm{H} \cdots A$ & $D-\mathrm{H}$ & $\mathrm{H} \cdots A$ & $D \cdots A$ & $D-\mathrm{H} \cdots A$ \\
\hline $\mathrm{C} 6-\mathrm{H} 6 \cdots \mathrm{O}^{\mathrm{i}}$ & 0.98 & 2.35 & $3.314(3)$ & 168 \\
$\mathrm{C} 11-\mathrm{H} 11 \cdots \mathrm{O} 1^{\mathrm{ii}}$ & 0.93 & 2.49 & $3.364(3)$ & 157 \\
\hline
\end{tabular}

Symmetry codes: (i) $x, y-1, z$; (ii) $-x+1, y+1 / 2,-z+1 / 2$. 